\title{
DIFICULDADES DA REDE DE SAÚDE MENTAL E AS REINTERNAÇÕES PSIQUIÁTRICAS: PROBLEMATIZANDO POSSÍVEIS RELAÇÕES*
}

Déborah Karollyne Ribeiro Ramos', Jacileide Guimarães², Simone Karine da Costa Mesquita ${ }^{3}$

${ }^{1}$ Enfermeira. Mestre em Enfermagem. Faculdade de Ciências Médicas de Campina Grande. Campina Grande-PB-Brasil. ${ }^{2}$ Enfermeira. Doutora em Enfermagem Psiquiátrica e Saúde Mental. Universidade Federal do Rio Grande do Norte. Natal-RN-Brasil. ${ }^{3}$ Enfermeira. Mestre em Enfermagem. Prefeitura Municipal de São Gonçalo do Amarante. Parnamirim-RN-Brasil.

RESUMO: Objetivou-se identificar dificuldades existentes na rede de saúde mental no Rio Grande do Norte, Brasil e problematizar possíveis relações entre essas e as reinternações psiquiátricas no estado. Realizou-se pesquisa exploratório-descritiva em abordagem qualitativa, cujo corpus originou-se da transcrição de entrevistas semiestruturadas, aplicadas a 20 trabalhadores de um hospital psiquiátrico de referência estadual, entre junho e agosto de 2011. Os resultados foram analisados por meio de análise de conteúdo temática. As categorias analisadas neste artigo foram: funcionamento inadequado dos serviços extra-hospitalares; dificuldade de acesso aos serviços substitutivos e a problemática da dependência química no contexto da atenção psicossocial. Conclui-se que as problemáticas evidenciadas se configuram como desafios contemporâneos para a saúde mental e expressam a necessidade de (re)visão da rede de saúde mental.

DESCRITORES: Saúde mental; Pesquisa nos serviços de saúde; Reforma dos serviços de saúde.

\section{DIFFICULTIES IN THE MENTAL HEALTH NETWORK AND PSYCHIATRIC READMISSIONS: DISCUSSING POSSIBLE RELATIONSHIPS}

\begin{abstract}
The aim of this study was to identify difficulties existing in the mental health services of Rio Grande do Norte, Brazil and to discuss possible relationships between these and psychiatric readmissions in the state. An exploratory-descriptive, qualitative study was conducted, the corpus of which originated from the transcription of semi-structured interviews, applied with 20 workers of a state reference psychiatric hospital, between June and August 2011. The results were analyzed using thematic content analysis. The categories analyzed in this article are: inadequate operation of outpatient services; difficult access to the substitute services, and the problem of chemical dependency in the context of psychosocial care. It was concluded that the problems highlighted are configured as contemporary challenges for mental health and express the need for a (re)view of the mental health network.
\end{abstract}

KEYWORDS: Mental health; Research in health services; Reform of health services.

\section{DIFICULTADES DE LA RED DE SALUD MENTAL Y LAS REINTERNACIONES PSIQUIÁTRICAS: PROBLEMATIZANDO POSIBLES RELACIONES}

RESUMEN: Se objetivó identificar dificultades existentes en la red de salud mental en Rio Grande do Norte, Brasil, y problematizar posibles relaciones entre esas y las reinternaciones psiquiátricas en el estado. Se realizó investigación exploratória y descriptiva en abordaje cualitativo, cuyo corpus resultó de la transcripción de entrevistas semiestructuradas, aplicadas a 20 trabajadores de un hospital psiquiátrico de referencia estadual, entre junio y agosto de 2011. Los resultados fueron analizados por medio de análisis de contenido temático. Las categorías analizadas en este artículo fueron: funcionamiento inadecuado de los servicios extra hospitalares; dificultad de acceso a los servicios sustitutivos y la problemática de la dependencia química en el contexto de la atención psicosocial. Se concluye que las problemáticas evidenciadas se configuran como desafíos contemporáneos para la salud mental y expresan la necesidad de (re)visión de la red de salud mental.

DESCRIPTORES: Salud mental; Investigación en los servicios de salud; Reforma de los servicios de salud.

*Artigo resultante de dissertação de mestrado apresentada ao Programa de Pós-Graduação em Enfermagem. Universidade Federal do Rio Grande do Norte, 2011. 


\section{INTRODUÇÃO}

A Reforma Psiquiátrica Brasileira (RPB) buscou (e ainda busca) desconstruir o tratamento cronificador e iatrogênico destinado ao paciente psiquiátrico. Trata-se de um movimento em prol da desinstitucionalização da assistência em saúde mental, com ênfase no tratamento extrahospitalar e da inclusão social da pessoa com transtorno mental ${ }^{(1)}$. Dessa forma, para garantir o suporte adequado ao indivíduo que apresenta transtorno mental e que se encontra em processo de desinstitucionalização foi criada, no Brasil, uma série de serviços substitutivos que devem funcionar de forma articulada e pautada na assistência em território. Os Centros de Atenção Psicossocial (CAPS), os Serviços Residenciais Terapêuticos (SRT), os Centros de Convivência e Cultura e o Programa De volta Para Casa, aliados as leis e portarias ministeriais, representam essa nova rede de atenção à saúde mental.

A rede territorial de assistência busca fomentar ações de promoção da saúde, prevenção e intervenção precoce em saúde mental, buscando o cumprimento da meta de internação como último recurso terapêutico, evitando, assim, a reinternação psiquiátrica ${ }^{(2)}$. No panorama atual da RPB observamos o emergir de dificuldades que são potencialmente opositoras à eficiência do processo de desinstitucionalização: a incompatibilidade entre a estrutura dos programas e serviços substitutivos de saúde mental e as necessidades habituais dos usuários desses dispositivos; o despreparo da comunidade para receber o indivíduo egresso de instituição psiquiátrica, uma vez que, quase todos os esforços são concentrados na mudança de comportamento dos usuários e quase nenhum para a modificação da mentalidade preconceituosa da sociedade; e, principalmente, os novos pacientes crônicos, cuja cronicidade foi adquirida por falhas na rede territorializada de serviços, culminando em internações e reinternações subsequentes desnecessárias ${ }^{(3)}$.

Diante desse contexto, há uma reflexão interessante: apesar de não compor a rede territorial de assistência ao sofrimento psíquico, o hospital psiquiátrico sofre os reflexos das dificuldades vivenciadas pelos dispositivos substitutivos. Pode-se supor que seja, provavelmente, por causa de tais problemáticas que a instituição psiquiátrica ainda tenha participação significativa na assistência ao transtorno mental, mesmo dentro de uma proposta de atenção psicossocial.

No estado brasileiro Rio Grande do Norte (RN), pesquisa aponta para a redução do quantitativo de reinternações psiquiátricas e para a diminuição na demanda por atendimento na urgência de um hospital psiquiátrico de referência estadual. Entretanto, ainda constata-se a persistência de índices insatisfatórios de reinternações psiquiátricas - 57,7\% no segundo semestre de 2011 - o que aponta para a representatividade da instituição psiquiátrica, no cotidiano da saúde mental do cenário estudado ${ }^{(4)}$. Os achados da referida pesquisa alertam para a persistência das reinternações psiquiátricas no cotidiano dos serviços de saúde mental. Tal fato merece atenção, visto que as reospitalizações em psiquiatria se configuram como um alerta epidemiológico para a necessidade de acompanhamento das mudanças nas práticas assistenciais e que hospitalizações frequentes podem gerar a cronificação da doença, aumento do isolamento e distanciamento do mundo real, privação do convívio familiar e social e fixação de estigmas ${ }^{(5-6)}$.

Essas observações preliminares instigam o seguinte questionamento: existem aspectos, na realidade da rede territorial de atenção ao sofrimento psíquico, que podem estar contribuindo para a persistência das reinternações psiquiátricas no estado do Rio Grande do Norte?

A presente pesquisa se justifica mediante a relevância científica e social da efetivação da rede de atenção à saúde mental. Além disso, a exigência ética de se desinstitucionalizar a assistência em saúde mental, bem como a necessidade do fortalecimento de novos dispositivos terapêuticos conferem importância ao estudo. Outro ponto a ser mencionado é o nosso esforço de sistematização, atualização e reflexão crítica sobre a problemática relação entre a rede territorial de atenção ao sofrimento psíquico, hospital psiquiátrico e reinternação. Deste modo, o estudo teve por objetivo identificar dificuldades existentes na rede de saúde mental do RN e problematizar possíveis relações entre essas e a realidade das reinternações psiquiátricas no estado.

\section{MÉTODO}

Trata-se de uma pesquisa com abordagem qualitativa e enfoque exploratório-descritivo, realizada em um hospital psiquiátrico do Sistema 
Único de Saúde (SUS), nos meses de junho e agosto de 2011. O hospital foi escolhido como cenário de estudo, pois além de ser referência em psiquiatria para o $\mathrm{RN}$, os profissionais que trabalham na instituição prestam assistência aos usuários em sofrimento psíquico, evidenciando, em seu cotidiano, os processos de admissão e alta hospitalar. Tal fato dota-os de uma visão abrangente sobre os usuários, familiares e o próprio funcionamento dos dispositivos substitutivos, aos quais estão cadastrados os usuários que recorrem à internação psiquiátrica.

Por tratar-se de uma pesquisa de abordagem qualitativa, a amostra foi selecionada de maneira não-probabilística e por acessibilidade a partir de uma população de 173 profissionais. Os critérios de inclusão para a amostra foram: ter começado a exercer atividades no referido hospital a partir de abril de 2001, período de regulamentação da Lei Federal 10.216/2001(7) que viabilizou a implantação da Política Nacional de Saúde Mental; e atuar na porta de entrada e/ou de saída do hospital regulando os processos de admissão e/ou de alta dos pacientes. Foi excluído do estudo o participante que, após a assinatura do Termo de Consentimento Livre e Esclarecido, optasse por não responder as perguntas presentes no instrumento de coleta de informações ou, ainda, aquele que decidisse retirar sua participação por livre e espontânea vontade.

Empregou-se a entrevista semiestruturada e a análise de informações do livro de registro de altas e internações (disponível no Serviço de Atendimento Médico e Estatístico da instituição lócus da pesquisa), correspondentes ao período de 2008 até o primeiro semestre de 2011, a fim de problematizar dados sobre as reinternações psiquiátricas.

A entrevista semiestruturada combina perguntas fechadas e perguntas abertas, possibilitando ao entrevistado discorrer sobre o tema proposto ${ }^{(8)}$. Partiu-se de um roteiro temático contendo questões envolvendo o conhecimento do profissional entrevistado sobre a rede de assistência à saúde mental do RN, o entendimento dos sujeitos sobre as prováveis causas das reinternações psiquiátricas na instituição - cenário da pesquisa - e sobre o impacto dos novos dispositivos de atenção à saúde mental nas reinternações psiquiátricas no hospital estudado. As entrevistas foram realizadas em local e horário previamente agendados de acordo com a disponibilidade e preferência dos sujeitos da pesquisa, buscando-se respeitar a privacidade do participante e a tranquilidade do ambiente.

Para análise dos dados coletados utilizouse a técnica de análise temática ${ }^{(8)}$, para tanto, os procedimentos pós-entrevista adotados foram divididos em quatro etapas, a saber: 1) transcrição na íntegra das gravações; 2) leitura e conferência do material; 3) envio do texto ao depoente para correção; 4) análise temática. De tal modo, realizou-se a leitura flutuante do material transcrito para se deixar impregnar pelo conteúdo das entrevistas. A familiaridade com este conteúdo possibilitou extrair as palavraschave, delimitar os contextos de compreensão das unidades de registro, recortar as falas e agrupá-las em categorias de análise. Com o agrupamento semântico das falas dos sujeitos foi possível identificar, partindo da perspectiva dos sujeitos, as principais dificuldades vivenciadas pelos serviços de saúde mental e que podem estar relacionadas às reinternações psiquiátricas no estado.

Em atendimento ao sigilo da identidade dos profissionais entrevistados neste estudo, denominaram-se os sujeitos da presente pesquisa com nomes de estrelas, em uma alusão à função dos corpos celestes. Ressalta-se que foram obedecidos os critérios éticos e legais dispostos nas Normas para Pesquisas Envolvendo Seres Humanos vigentes ${ }^{(9)}$. O projeto de pesquisa foi encaminhado para apreciação do Comitê de Ética em Pesquisa da Universidade Federal do Rio Grande do Norte sob o protocolo n. 019/11, recebendo aprovação desta instância no dia 15 de junho de 2011, através do parecer n. 216/2011 e CAAE 0021.0.051.000-11.

\section{RESULTADOS}

Foram entrevistados 20 profissionais de saúde de um hospital psiquiátrico, sendo 14 mulheres e seis homens. As categorias profissionais foram: seis psiquiatras, dois psicólogos, um terapeuta ocupacional, dois assistentes sociais e nove técnicos de enfermagem.

A análise do material empírico permitiu identificar as seguintes categorias relacionadas às principais dificuldades, vivenciadas pela rede territorial, na atenção ao paciente em sofrimento 
psíquico: funcionamento inadequado dos serviços extra-hospitalares; dificuldade de acesso dos usuários aos serviços substitutivos; a problemática da dependência química no contexto da atenção psicossocial; dificuldade de usuários, familiares cuidadores e profissionais em aderir aos dispositivos da rede de saúde mental; desconhecimento de parcela dos profissionais da instituição sobre a rede de atenção à saúde mental. De acordo com os participantes da pesquisa estes fatores foram apontados como aspectos que contribuem para as reinternações psiquiátricas no estado.

Salienta-se que, neste artigo, são analisadas as três primeiras categorias devido ao espaço disponibilizado para a apresentação e discussão dos resultados. As demais categorias apontadas serão abordadas em recortes futuros.

\section{Funcionamento inadequado dos serviços extra- hospitalares}

Com relação ao funcionamento dos dispositivos substitutivos, encontram-se argumentações apontando a falta de psiquiatras nos serviços, especialmente no Centro de Atenção Psicossocial - CAPS III, como uma das grandes dificuldades enfrentadas pela rede de saúde mental do RN, segundo os recortes abaixo:

Eu acho que o CAPS III aqui em Natal é um modelo que não tem psiquiatra 24 horas, só tem 12 horas, as outras 12 horas da noite os pacientes ficam com a equipe de enfermagem somente e com sobreaviso do SAMU (Serviço de Atendimento Móvel de Urgência). Se o SAMU tiver numa emergência lá do outro lado da cidade e se tiver uma emergência no CAPS naquele momento, é impossível ele estar em dois lugares ao mesmo tempo. Quantidade de leitos abertos: 8 leitos, 6 leitos. Irrisório pra quantidade de pacientes. (Arcturus)

A questão é a falta de funcionamento adequado dos CAPS que a gente não encontra o profissional psiquiatra de segunda à sexta de plantão, como de fato ele deveria estar. E daí, quando realmente acontece um surto, acontece uma urgência, $o$ local que eles [usuários] procuram realmente éo hospital, porque tem psiquiatra 24 horas. (Alrisha)
Ainda, a respeito do funcionamento dos serviços extra-hospitalares e considerando a participação do SAMU no atendimento às urgências psiquiátricas, encontram-se discursos como os seguintes:

Eu acho que a importância do psiquiatra no SAMU seria pra tentar resolver os problemas que pudessem ser resolvidos e esses pacientes não virem pra cá, certo? Não percebo isso. Continua vindo a quantidade muito grande do SAMU pra cá. (Arcturus)

O SAMU ele costuma trazer muitos pacientes. Então, às vezes, até pacientes que, a nosso ver $e$ ao ver da equipe, que não haveria necessidade de um atendimento de pronto socorro [psiquiátrico] naquele momento. (Algol)

\section{Dificuldade de acesso dos usuários aos serviços substitutivos}

Sobre as dificuldades de usuários e familiares em acessar os serviços substitutivos da rede de saúde mental do estado, refere-se:

A gente sabe da dificuldade, principalmente na Zona Norte da capital, de se conseguir um atendimento médico. A gente sabe dessa dificuldade dos pacientes ao saírem daqui e darem continuidade ao seu tratamento. (Auva)

Há que se reconhecer o comprometimento que a dificuldade de acesso aos serviços substitutivos pode ter no tratamento dos usuários de saúde mental, tendo em vista que a interrupção do acompanhamento pode culminar em internação do usuário, como mostra as falas de Mizar e Shaula:

Ospacientesqueconseguemumacompanhamento pós-alta realmente têm uma taxa de reinternação menor, o problema está sendo o acesso desses pacientes ao serviço. (Mizar)

Muitas vezes o paciente demora pra conseguir uma consulta na rede. Às vezes, passa numa triagem, mas quando ele vai passar por um psiquiatra demora meses e não dá pra o paciente esperar. Muitas vezes, você acaba internando 
porque o paciente vai piorar se não tiver um acompanhamento logo. (Shaula)

Dessa maneira, há o uso da internação, que deveria ser utilizada apenas em último caso, como uma forma de prevenção de agravos à condição de doente mental. Tal atitude se configura como um viés que potencializa riscos aos princípios conquistados do SUS e da RPB, podendo configurar-se em um retorno indiscriminado, embora se diga bem intencionado, às soluções manicomiais.

\section{A problemática da dependência química no contexto da atenção psicossocial}

Outra situação-problema apresentada pelos sujeitos da pesquisa refere-se ao atendimento da dependência química, como uma demanda da rede de saúde mental que, por suas peculiaridades, dificulta a inserção social de usuários e contribui para as estatísticas de reinternações psiquiátricas.

Hoje em Natal, não só em Natal como no Rio Grande do Norte, a demanda de droga é um problema de saúde pública [...] porque a droga está atingindo até o que tem transtorno mental, ele está se tornando paciente que tem transtorno mental associado com a droga [...] o quadro dele vai se complicar de tal forma que não vai ter mais o controle das crises que se poderia ter. (Mira)

Em um contexto ampliado, a dependência química pode exacerbar o quadro psíquico e neurológico da pessoa que apresenta sintomatologia psiquiátrica, concorrendo, assim, para futuras internações/reinternações psiquiátricas. Além disso, o comprometimento da intersetorialidade e da implementação (in) satisfatória de políticas de enfrentamento da dependência química podem contribuir, sobremaneira, para tais achados.

\section{DISCUSSÃO}

Como se vê nos recortes das falas, a falta de psiquiatras nos serviços substitutivos foi apontada como um dos fatores contribuintes para a inadequação do funcionamento da rede territorial de assistência ao sofrimento psíquico, culminando no favorecimento das reinternações psiquiátricas. A este respeito argumenta-se sobre o ônus de se manter um serviço tipo CAPS III, assegurando nele a permanência do profissional psiquiatra durante as 24 horas do dia e os sete dias da semana, dificuldade essa relacionada ao custo financeiro e às próprias condições do mercado reduzido de médicos especializados em psiquiatria $^{(10)}$.

Arcturus (participante da pesquisa) comenta sobre o risco de se deixar um serviço tipo CAPS III, que deve ser apto a receber casos de urgência psiquiátrica, sem a permanência do psiquiatra. Fazendo uma reflexão minuciosa e honesta sobre o atendimento de psiquiatria, sabe-se que ter psiquiatra presente, por si só, não é garantia de assistência. $\mathrm{Na}$ atualidade, como disse o sujeito da pesquisa, ter SAMU de sobreaviso também não é garantia de assistência, pois se acontecer duas urgências simultâneas o atendimento à solicitação do CAPS III fica ao crivo do médico regulador do SAMU, o qual por herança sociocultural ou paradigmática, conforme estudo realizado na realidade local ${ }^{(11)}$, tem preterido a urgência psiquiátrica por achar que essa é de pouca importância frente a outras clínicas, como a clínica médica ou trauma.

Por outro lado, não se deve atribuir ao psiquiatra toda a responsabilidade com os atendimentos efetuados em saúde mental. É preciso problematizar a presença desse profissional nos serviços, levando em consideração os preceitos da RPB como a descentralização da assistência em saúde mental como prevista na portaria no 336/2002 ${ }^{(12)}$ e a existência dos técnicos de referência (ou terapeutas de referência) que devem estar aptos a acolher e acompanhar os usuários ${ }^{(13)}$.

Sobre as possibilidades advindas da existência do terapeuta de referência dentro dos serviços territoriais de saúde mental, autores brasileiros ${ }^{(14)}$ salientam dificuldades vivenciadas por serviços e profissionais da área. Por exigir uma articulação entre diversos saberes e entre vários campos relacionais (familiar, laboral, social, cultural), a atuação do técnico de referência suscita mudanças no modo de trabalho desenvolvido pelos profissionais. Transformações essas, voltadas para a reinvenção da maneira de ser e de agir dos trabalhadores no desenvolvimento das ações de 
cuidado para com o usuário dos serviços de saúde mental ${ }^{(14)}$. Talvez o despreparo das equipes para atuar na perspectiva da atenção psicossocial, tendo por base o técnico de referência, poderá estar contribuindo para a dependência, por parte dos serviços, da presença do psiquiatra para acolhimento dos usuários que chegam à unidade.

Outro ponto abordado nas falas dos sujeitos da pesquisa foi à dependência química no contexto da atenção psicossocial como uma realidade problemática da rede de saúde mental e que traz contribuições para as reinternações. A esse respeito, estudo realizado no hospital pesquisado comprova a representatividade da dependência química na demanda do pronto socorro da instituição. Dados recentes mostram aumento de $0,23 \%$ dos atendimentos para alcoolistas e drogadictos, por um lado, e a redução de 0,98\% dos atendimentos aos esquizofrênicos por outro ${ }^{(4)}$.

Com efeito, não podemos desconsiderar o potencial decorrente do uso de drogas para o desenvolvimento de transtornos mentais nos indivíduos, fato que pode contribuir para o aumento de casos de transtornos psiquiátricos na comunidade e, assim, o aumento nos índices de primeiras internações. Dentro dessa temática, lembrando que o uso abusivo de drogas lícitas ou ilícitas é um problema que assola não apenas o RN, mas o Brasil como um todo, questionase o preparo da rede de saúde para atender a este público específico que demanda tantas peculiaridades.

A complexidade da atenção à problemática de álcool e outras drogas, divulgada com maior propriedade a partir da experiência concreta dos CAPSad (Centro de Atenção Psicossocial Álcool e Drogas), revela a exigência cotidiana de implementação da inven-tividade, da multiplicação de iniciativas e da intersetorialidade dentro desta nova perspectiva assistêncial ${ }^{(10)}$.

Considerando a relevância de um trabalho intersetorial para o enfrentamento da dependência química, conclusões de estudo realizado em Maringá apontam, de forma geral, para um grau insatisfatório de implantação de diretrizes para enfrentamento do álcool e outras drogas na Atenção Primária à Saúde no município. Alegase, ainda, a falta de comunicação entre Estratégia de Saúde da Família e o CAPSad de referência. Diante dos achados, os autores concluem sobre a necessidade de aperfeiçoamento da atenção à Saúde Mental e para a importância da análise do papel dos diferentes equipamentos de saúde que compõem a rede de saúde, ponderando que a atenção integral é constituída por um conjunto de dispositivos intersetoriais ${ }^{(15)}$.

As conclusões do estudo, exposto acima, fazem refletir sobre uma provável relação entre a dependência química no atual contexto da atenção psicossocial, a problemática da persistência das reinternações psiquiátricas e a intersetorialidade das ações de promoção da saúde e de redução de danos. Acredita-se que essa é uma temática que suscita mais pesquisas que proponham mecanismos para envolvimento de diversos setores da área da saúde, assim como de usuários, familiares e profissionais, contribuindo para o manejo adequado do sofrimento psíquico e da dependência química, na perspectiva da manutenção de uma assistência em território para esse público.

Pôde-se inferir que as dificuldades debatidas podem camuflar o êxito parcial que vem sendo conquistado pela saúde mental do $\mathrm{RN}$, além de contribuírem para a existência de índices ainda elevados de reinternações psiquiátricas no estado, configurando-se como desafios, alguns já clássicos, outros emergentes, na atualidade da saúde mental nacional e estadual.

\section{CONSIDERAÇÕES FINAIS}

Esse estudo trouxe à tona dificuldades vivenciadas pelos serviços de saúde mental do estado e que, até então, eram percebidas por usuários e profissionais apenas pela observação assistemática.

É sabido da grande dificuldade em encontrar alternativas para resolver velhos e novos problemas, mas acredita-se que um fator em especial perpassa todos esses pontos: o diálogo e a articulação efetiva entre todos os dispositivos que compõem a rede de atenção à saúde mental.

Destaca-se a validade e a relevância da presente pesquisa pelo fato de ter se debruçado sobre a realidade de profissionais, que atuam na assistência direta de uma instituição de referência estadual, para o atendimento em psiquiatria. Apesar da não pretensão de esgotar as discussões sobre a temática, a síntese interpretativa das 
informações coletadas nessa pesquisa podem contribuir para comparações e novas discussões sobre o assunto em pauta.

Acredita-se que os achados desse estudo expressam necessidade de uma (re)visão por parte de profissionais, gestores, usuários, familiares e comunidade, em geral, acerca de temas que devem ser colocados em pauta para uma "nova agenda" de discussão, reorganização e inventividade da rede de saúde mental do estado, do Brasil e dos demais países do mundo que buscam desenvolver uma atenção integral e de cunho psicossocial ao sofrimento psíquico.

Como principal limitação, do presente estudo, coloca-se a redução do cenário da pesquisa a um único hospital psiquiátrico, fato que pode ter culminando em uma visão restrita do fenômeno estudado. Entretanto, na tentativa de minimizar possíveis vieses optamos por realizar o estudo em uma instituição referência estadual para o atendimento em psiquiatria.

A produção de conhecimento referente à temática em foco e a troca de saberes entre profissionais da área favorecem um cuidado integral e contínuo em busca de promoção a saúde, respeitando a integridade, equidade e cidadania, como também, contribuem para superação do estigma e segregação das pessoas que vivenciam os sofrimentos psíquicos.

\section{REFERÊNCIAS}

1. Amarante P. Reforma Psiquiátrica e Epistemologia. Cad. bras. saúde mental. [Internet] 2009;1(1) [acesso em 26 jun 2014] Disponível: http://www.cbsm.org.br/artigos/ artigos/04_paulo_amarante.pdf.

2. Ministério da Saúde (BR). Portaria n. 3.088, de 23 de dezembro de 2011. Institui a Rede de Atenção Psicossocial para pessoas com sofrimento ou transtorno mental, incluindo aquelas com necessidades decorrentes do uso de crack, álcool e outras drogas, no âmbito do Sistema Único de Saúde (SUS). Brasília: Ministério da Saúde; 2011.

3. Desviat M. A Reforma Psiquiátrica. 2a reimp. Rio de Janeiro: Fiocruz; 2008.

4. Ramos DKR, Guimarães J. Novos serviços de saúde mental e o fenômeno da porta giratória no Rio Grande do Norte. Reme. [Internet] 2013;17(2) [acesso em 12 fev 2014] Disponível: http://www.reme.org.br/artigo/ detalhes/661.
5. Consoli GL, Hirdes A, Costa JSD. Saúde mental nos municípios do Alto Uruguai, RS, Brasil: um diagnóstico da reforma psiquiátrica. Ciênc. saúde colet. [Internet] 2009;14(1) [acesso em 12 fev 2014] Disponível: http:// dx.doi.org/10.1590/S1413-81232009000100017

6. Pinheiro TLS, Cazola LHO, Sales CM, Andrade ARO. Fatores relacionados com as reinternações de portadores de esquizofrenia. Cogitare enferm. [Internet] 2010;15(2) [acesso 12 fev 2014] Disponível: http://ojs.c3sl.ufpr.br/ojs2/index.php/cogitare/article/ view/17865/11657

7. Ministério da Saúde (BR). Lei n. 10.216, de 6 de abril de 2001. Dispõe sobre a proteção e os direitos das pessoas portadoras de transtornos mentais e redireciona o modelo assistencial em saúde mental. Brasil: Ministério da Saúde; 2001.

8. Minayo MCS. O desafio do conhecimento: pesquisa qualitativa em saúde. $7^{a}$ ed. São Paulo: Hucitec; Rio de Janeiro: Abrasco; 2000.

9. Ministério da Saúde (BR). Conselho Nacional de Saúde. Diretrizes e normas regulamentadoras de pesquisa envolvendo seres humanos. Resolução n. 196, de 10 de outubro de 1996. Brasília; 1996.

10. Tenório F. Questões para uma atualização da agenda da Reforma Psiquiátrica. In: Couto MCV, Martinez RG (organizadores). Saúde Mental e Saúde Pública: questões para uma agenda da Reforma Psiquiátrica. Rio de Janeiro: Núcleo de Pesquisa em Políticas Públicas e da Saúde Mental; 2007. p. 13-28. [Internet] [acesso 29 mai 2014]. Disponível: www.nuppsam. org/page5.php

11. Bonfada D. Serviço de Atendimento Móvel de Urgência (SAMU) e as urgências psiquiátricas [dissertação]. Natal (RN): Universidade Federal do Rio Grande do Norte; 2010. [Internet] [acesso 29 mai 2014]. 147 p. Disponível: http://repositorio.ufrn.br:8080/jspui/ bitstream/1/9273/1/DiegoB_DISSERT.pdf.

12. Ministério da Saúde (BR). Portaria GM n. 336, de 19 de Fevereiro de 2002. Institui as diferentes modalidades dos CAPS. Brasília: Ministério da Saúde; 2002.

13. Ministério da Saúde (BR). Secretaria de Atenção à Saúde. Departamento de Ações Programáticas Estratégicas. Saúde mental no SUS: os centros de atenção psicossocial. Brasília: Ministério da Saúde; 2004. [Internet] [acesso 12 fev 2014]. Disponível: http://portal.saude.gov.br/portal/arquivos/pdf/manual_ caps.pdf.

14. Silva EA, Costa II. O profissional de referência em Saúde Mental: das responsabilizações ao sofrimento psíquico. Rev. latinoam. psicopatol. fundam. [Internet] 2010;13(4) [acesso em 29 mai 2014] Disponível: http:// dx.doi.org/10.1590/S1415-47142010000400007 
15. Santos JAT, Oliveira MLF. Implantação de ações para enfrentamento do consumo de drogas na atenção primaria à saúde. Cogitare enferm. [Internet] 2013;18(1) [acesso 12 fev 2014] Disponível: http://ojs.c3sl.ufpr.br/ ojs2/index.php/cogitare/article/view/28977/20009 